OPEN ACCESS

Edited by: Abdulkadir Celik,

King Abdullah University of Science and Technology, Saudi Arabia

Reviewed by:

Boya Di,

Imperial College London, United Kingdom

Hajar El Hammouti,

King Abdullah University of Science and Technology, Saudi Arabia

*Correspondence:

Harri Saarnisaar harri.saarnisaari@oulu.fi

Specialty section: This article was submitted to

Wireless Communications,

a section of the journal

Frontiers in Communications and

Networks

Received: 17 May 2021

Accepted: 28 October 2021

Published: 30 November 2021

Citation:

Saarnisaari $H$, Chaoub A, Heikkilä M, Singhal A and Bhatia V (2021) Wireless

Terrestrial Backhaul for 6G Remote

Access: Challenges and Low

Power Solutions.

Front. Comms. Net 2:710781.

doi: 10.3389/frcmn.2021.710781

\section{Wireless Terrestrial Backhaul for 6G Remote Access: Challenges and Low Power Solutions}

\author{
Harri Saarnisaari ${ }^{1 *}$, Abdelaali Chaoub ${ }^{2}$, Marjo Heikkilä ${ }^{3}$, Amit Singhal $^{4}$ and Vimal Bhatia ${ }^{5}$ \\ ${ }^{1}$ Centre for Wireless Communications, University of Oulu, Oulu, Finland, ${ }^{2}$ STRS Laboratory, INPT, Rabat, Morocco, ${ }^{3}$ Centria \\ University of Applied Sciences, Ylivieska, Finland, ${ }^{4}$ Department of Electronics and Communications Engineering, Bennett \\ University, Greater Noida, India, ${ }^{5}$ Centre for Advanced Electronics, IIT Indore, Indore, India
}

Despite developments in communication systems over the last few decades, a digital divide exists in the unconnected part of the world. The latter is characterized by large distances to internet access points, underdeveloped infrastructure, sparse populations, and low incomes. This concern of digital divide is raised in the sixth generation's (6G) initial vision as an extremely important topic. However, it is important to understand affiliated challenges and potential solutions to achieve this vision. Motivated by the recent backhaul link forecasts that expect a dominance of the microwave technology within the backhauling market, this paper studies the potential of a low-power terrestrial microwave backhaul from the sufficient-data-rate and solar powering perspective. Competing technologies (e.g., fiber) may not be energy efficient and commercially viable for global connectivity. Since rural and remote areas may not have grid power, we look at the viability of alternative sustainable sources, in particular solar power, to power the wireless backhaul in 6G. In addition, we also explore services for the operators and users to use the system efficiently. Since the access points are connected to backhaul, we also compare the two prominent solutions based on low-power small-radius cells and a mega-cell that covers a large area and show insights on the power autonomy of the systems. In the end, we propose directions for research and deployment for an inclusive connectivity as a part of future $6 \mathrm{G}$ networks.

Keywords: unconnected, under-connected, rural, connectivity, wireless, digital oasis

\section{INTRODUCTION}

Without a doubt, the digital divide between urban and remote/rural areas is a problem in our societies even in the 21 st century. The unconnected or under-connected people often live in rural and remote areas characterized by low population density, low income or poverty, difficult terrain, and nonexistent or unreliable grid power supply. Nonavailability of any one of these basic conditions means reduced willingness to invest in the communication and connectivity infrastructure by commercial operators. This has been addressed in many places; however, Handforth (2019) expressed this concern in detail. In addition, the COVID-19 pandemic has recently raised interest in rural and remote areas as potential places of working instead of urban areas. However, many of the aforementioned characteristics remain.

The sixth generation $(6 \mathrm{G})$ of wireless networks could reach a major milestone and be the first mobile generation where solutions to reduce the digital divide are benefiting for more consideration 
since the early research efforts. For this reason, it is important to understand what the challenges are, before solutions can be proposed to the standardization bodies. Indeed, global coverage has been addressed in a big picture in 6G white papers such as those of Latva-aho and Leppänen (2019) and Saarnisaari et al. (2020) and in an initial project vision (Hexa-X, 2021). However, the focus in many $6 \mathrm{G}$ visions has been mainly on higher data rates rather than on connecting the unconnected. The digital divide is addressed by some organizations, and interesting information about connectivity status, challenges, requirements, and goals can be found in their reports. These organizations include the Broadband Commission for Sustainable Development that was launched by the International Telecommunication Union (ITU) and the United Nations Educational, Scientific and Cultural Organization (UNESCO) in 2010; IEEE Future Networks; the GSM Association (GSMA); the connectivity task forces of the Arctic Council; and the 6G Flagship, which provided the above-mentioned white papers.

One important factor in the connectivity solution is the backhaul connection or a link between the internet and the local access point. An appropriate backhaul design is especially challenging in the mentioned remote areas. Emerging satellite constellations such as Starlink by SpaceX and the systems by OneWeb and Telesat can be game-changers once operational. However, their affordability and sustainability (in the economic sense) are still of major concern. On one hand, optical fibers play a significant role in the terrestrial backbone, but on the other hand, widespread planning and digging for laying fibers are not always feasible. Furthermore, the communication infrastructure required to realize this is absent in many countries. For example, the ratio of deployed fiber to the total population is merely 0.1 in India compared with 1.2 in the United States as discussed by Khaturia et al. (2020). The forthcoming 6G networks are expected to leverage the power of flying platforms [e.g., unmanned aerial vehicles (UAVs) and high-altitude platform stations (HAPSs)] in different network segments including the backhaul part, especially in harsh terrains or if terrestrial deployments have collapsed or are absent. To achieve this goal, non-terrestrial networks (NTNs) need to address a number of technical impediments related to their integration with terrestrial infrastructure, their placement in three-dimensional space, and their energy efficiency concerns, thus calling for further research. Another recent trend is the use of free space optical (FSO) links to expand coverage and capacity in shadowed locations using the license-free optical bands. However, despite these advances, there are still various technical hurdles including those related to sensitivity to optical misalignment and atmospheric turbulence. On the other hand, new spectrum developments [e.g., transition to terahertz bands potentially supported by massive multiple input and multiple output (MIMO) and beamforming] hold great promise as a key driver for network capacity expansion. This is particularly true for indoor coverage, but not yet for outdoor and backhaul usage as a result of the luck of hardware [e.g., radio frequency (RF) integrated circuit technologies] maturity and affordability (Pärssinen et al., 2020).
While appealing research paradigms such as NTN and FSO have attracted an overwhelming interest as promising backhauling technologies to connect remote and rural regions, traditional wireless backhaul links have been largely overlooked in previous studies. Surprisingly, it is forecasted that microwave links will be the dominant backhaul technology within the next 6 years (from 2021 to 2027), accounting for approximately $65 \%$ of the global market share for both macro-cells and small cells according to a recent report (GSMA, 2021). Therefore, terrestrial microwave relay technology, which is currently the most popular backhaul solution, still remains a key option in the future. When the connectivity gap is considered, low cost, easy installation/ maintenance, and self-powered autonomous microwave relays (and access points) are elementary factors that have to be addressed in the research and development as discussed by Handforth (2019). An overview of the existing technologies is also provided by Yaacoub and Alouini (2020).

In the remote and rural areas, the backhaul has to operate in hard-to-access terrains and environments wherein critical infrastructure (e.g., power grid) might be missing or unreliable. Consequently, investments in these areas can be regarded as economically not viable for mobile network operators (MNOs). However, together with the local access, the backhaul has to offer affordable (usage cost) and sufficient (data rate) services to the people and facilities in the so-called digital oases, where people live and work, as pointed out by Chaoub et al. (2021) and Saarnisaari et al. (2020). As an example, an oasis can be a village, a farm, or a mine.

Wireless backhauling in dense networks and high-traffic conditions, i.e., urban environments, has been extensively studied in the literature. Fiber-based solution is seen as the most energy efficient in a comparison made by Tombaz et al. (2014) and Ge et al. (2014) from the energy efficiency perspective. While fiber plays an important role, microwave backhaul will account for the majority of global backhaul links from 2021 to 2027, with an approximately $65 \%$ market share (GSMA, 2021). A survey of $5 \mathrm{G}$ backhaul connectivity is provided by Jaber et al. (2016), and the backhaul component is recognized as the bottleneck for $5 \mathrm{G}$ and beyond-5G mobile generations. The integrated access and backhaul (IAB) paradigm for $5 \mathrm{G}$ mmWave is studied by Polese et al. (2020). Furthermore, a more historical overview focusing on circuit-switched networks is given by Tipmongkolsilp et al. (2011), and a more recent one that introduces the latest breakthrough technologies is given by Tezergil and Onur (2021).

The aim of this paper is to discuss challenges, stakeholders and their possible role, and more thoroughly, terrestrial backhaul requirements and potential solutions towards $6 \mathrm{G}$. Based on the review of the current state of the art, this paper is the first attempt to address the main opportunities and challenges associated with wireless terrestrial backhaul in remote and rural areas. A special emphasis is given to the trending solutions that remote relay links should embrace for substantial energy savings while maintaining sufficient capacity for the radio cells being served. As such, this work can be used as a springboard for further $6 \mathrm{G}$ discussions and definitions concerning rural and remote area solutions, especially related to terrestrial backhaul. This article considers cases where 


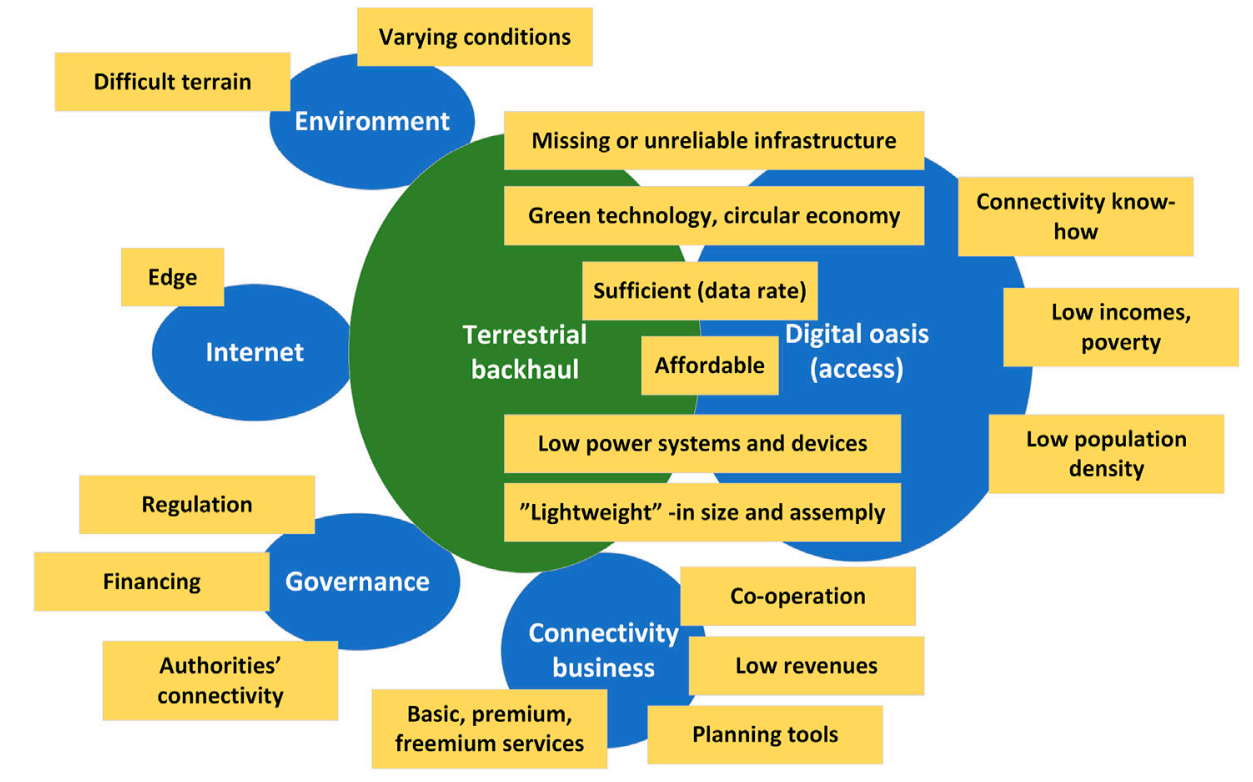

FIGURE 1 | Challenges in 6 G backhaul solutions for connecting the unconnected digital oasis.

the internet is a few hops away from the local access, excluding extremely remote and nearly empty (e.g., deserts, wilderness, seas, and airspace) areas. To this end, Figure 1 shows the challenges and stakeholders that will be discussed in more detail in the coming sections. As illustrated in the figure, the terrestrial backhaul and the access to the oasis share many common challenges.

In particular, we evaluated how much power is needed in microwave links with sufficient data rates in remote areas to see their feasibility for solar-powered operation in various places around the globe. Power consumption models of cellular towers are used in the analysis as these are the most commonly proposed and deployed. Our study reveals certain gaps between possibilities and current solutions and opens floors to our discussion about low-power remote/rural area solutions within 6G. Since the access point has to be connected to the backhaul and possibly it also has to be a low-power system and rely on the same power source, we analyzed the power needed in an example oasis and compared that with a mega-cell solution that could provide access to many oases using a single high tower. This shows the feasibility of mega-cells as $6 \mathrm{G}$ remote area solutions with less networking costs for connection to the backhaul, however at the expense of high energy consumption. Smaller and more compact cells, on the other hand, are clearly more energy efficient but many more are needed to cover the same area as one mega-cell and may need many backhaul links.

Based on the above introduction, the key contributions of this paper are threefold:

- Emphasizing the significant potential of terrestrial microwave backhauling as a key enabler for an inclusive and energy-efficient $6 \mathrm{G}$ connectivity. It has been shown that terrestrial microwave links can provide enough capacity to accommodate the traffic needs of end users in remote and rural places using reasonable solar energy systems. This is confirmed in the recent forecasts provided by GSMA (2021).

- Discussing (1) the challenges that hinder the evolution of the terrestrial microwave backhaul technology, (2) the current and the future popular use cases, (3) the involved stakeholders, and (4) the impact of the last-mile access (i.e., either low-power base station or mega-cell) on backhaul design in those isolated areas.

- Insightful comments and recommendations are provided along with the promising features and the open research questions related to evolving terrestrial microwave backhauling over the $6 \mathrm{G}$ era.

In order to address the above-mentioned issues, the paper is organized as follows. Section 2 presents the current low-cost terrestrial backhaul solutions that potentially rely on renewable energy. In addition, low-power access point and alternative megacell technology to cover several oases are discussed. Section 3 considers the operation environment and the challenges it creates. This discussion includes terrain, demographics, and data rate requirements. It is quite possible that "unlimited" backhaul is not accessible in these challenging environments. Therefore, in Section 3.2, we discuss solutions that people in the oasis could follow to utilize the possibly limited backhaul. This opens possibilities for innovation and cooperation between various players and disciplines. The potential roles of other stakeholders such as governance and connectivity business sectors are presented in Section 3.3. As our primary focus, deeper discussion about backhaul is provided in Section 4 where a gap between current low-energy solutions and theoretically possible solutions is discussed to show potential 
for innovation in $6 \mathrm{G}$. We analyze the required transmit power as a function of distance and data rate per radio link and highlight the power requirement in the backhaul towers. We also discuss potential low-power solutions towards $6 \mathrm{G}$ and compare megacell access with low power and low tower access. Finally, we provide a summary of proposed future research directions in Section 5.

\section{CURRENT STATUS}

This section provides an overview of the current low-power backhaul technologies, as well as low-power access solutions and the large-cell counterpart.

\subsection{Microwave Technology}

Microwave link technology is a mature business as shown, e.g., in Edstam et al. (2017), Ericsson (2018), and Ericsson (2020). It uses multiband technology, several carriers, up to $16 \mathrm{k}$ quadrature amplitude modulation (QAM), and adaptive modulation and coding to achieve over 1 Gbps data rates. Frequency bands in the 6-15 GHz range provide narrowband services, $18-42 \mathrm{GHz}$ widefrequency bands, and $70 / 80 \mathrm{GHz}$ very wide bands. It is expected that this technology will evolve and provide solutions that are suitable for affordable remote area solutions. Traditional microwave links can cover hundreds of kilometers, though they could also be used to boost capacity at shorter distances, for fixed wireless access. In this study, the focus is on an overview of lightweight, solar-powered solutions over shorter distances, since without a reliable power grid, renewable energy sources or diesel generators must be used. Refueling of diesel generators consumes a substantial part of operating expenses (OPEX) (Handforth, 2019); therefore, technology that allows us to minimize refueling would be greatly beneficial. In areas where sunlight is available year-round, solar energy could be sufficient as detailed below.

One example of power consumption for modern lightweight designs is the RAy microwave links by Racom (2021); e.g., RAy 3 consumes on average $22 \mathrm{~W}$ and provides -30 to $10 \mathrm{dBm}$ output power with data rates varying from $3.5 \mathrm{Mbps}$ to $1 \mathrm{Gbps}$ depending on modulation and coding scheme and the bandwidth operating at 17 and $24 \mathrm{GHz}$ frequencies. This design assumes that the towers are solar powered. However, it should be kept in mind that each relay station in the middle requires at least two links or operates in a half-duplex mode. Likewise, local access points require a backhaul link as well as the access system.

Another interesting approach is to apply Wi-Fi technology as in the Fraunhofer Wiback solution. ${ }^{1}$ It provides end-to-end quality-of-service provisioning, auto-configuration, selfmanagement, and self-healing, making operations easy. It utilizes IEEE 802.11ac technology and correspondingly provides up to $200 / 400 \mathrm{Mbps}$ data rates over $40 / 80 \mathrm{MHz}$ channel bandwidths with $20 \mathrm{~km}$ maximum link distance. The

${ }^{1}$ https://www.wiback.org/ latest version (v.4) comes with a built-in solar charger and a battery aiming to provide 24/7/365 operation with a solar panel of about $100 \mathrm{~W}$. The average power consumed is said to be $10 \mathrm{~W}$ and a maximum of $16 \mathrm{~W}$ to cover signal back-off. ${ }^{2}$ Indeed, Rademacher et al. (2013) claimed that it can achieve a $200 \mathrm{Mbps}$ one-way data rate over $10 \mathrm{~km}$ with this average power using a dish antenna with $24-30 \mathrm{dBi}$ gain (depending on carrier frequency).

\subsection{Access Technology}

Access points or base stations are connected with backhaul relay links but may also serve as relays to subsequent access points. Therefore, access technology plays an equally important role in the end-to-end solution. Furthermore, access principles affect the required backhaul towers and links. Since the emphasis of this work is on terrestrial solutions, we discuss two solutions based on low-power base stations and high tower (or spot)-based megacells, and later on, we analyze their differences and possible impact on backhaul.

Huawei's RuralStar series introduced in GSMA Connected Society (2019) is a good example of low-power access point technology. In particular, Huawei's RuralStar Pro solution, launched in February 2020, is a rural lightweight base station, leveraging the IAB concept by integrating baseband, RF, and wireless backhaul functions. It provides voice and mobile broadband services to remote regions with a population smaller than 500 and requires only two solar panels to power the entire site. The power consumption of the site is about $100 \mathrm{~W}$, which is far lower than that of a traditional site, and the platform can be installed on a $6 \mathrm{~m}$ pole, thus boosting the energy efficiency of the system and simplifying the installation costs. They claim that the first commercial sites provide interesting results such as the fact that the installation took only $2 \mathrm{~h}$, which is $80 \%$ less time than it takes to install a traditional site. In addition, after the site was deployed, the coverage proportion in the village increased from $5 \%$ to $85 \%$, and the population can enjoy LTE voice services and a data download speed of $20 \mathrm{Mbps}$.

An alternative solution exploiting the mega-cell concept was prototyped by Facebook Connectivity under the name SuperCell. The main insights from the project have been recently published in Bondalapati et al. (2020). This solution exploits tall towers (up to $250 \mathrm{~m}$ ) with narrow-sectored, high-gain antennas $(29 \mathrm{dBi})$ for wide coverage and high capacity, while being economically viable compared with the conventional macro-cell technologies under the same topography. Real field trials were conducted using a 12sector Luneburg lens antenna system over LTE Band 41 (i.e., $2.5 \mathrm{GHz}$ ). These experiments demonstrated a downlink throughput of up to $7.8 \mathrm{Mbps}$ and an uplink throughput of up to $1.2 \mathrm{Mbps}$ at a range of $40 \mathrm{~km}$. Seamless inter-sector handover was observed in the SuperCell deployment. A detailed economic analysis from the uncovered Nigerian regions showed that a single SuperCell could replace 15 to 25 traditional macro-cells, thus achieving the same performance of a traditional network of

${ }^{2}$ WIBACK NODE-2-CONNECT II DATASHEET: https:/www.wiback.org/ content/dam/wiback/en/documents/WiBACK_EN_N2C2_11-2017.pdf 
macro-cells at 33\% lower overall cost of ownership. However, this solution needs to overcome a number of technical hurdles before real-life deployments, including its high power consumption in addition to being potentially a single point of failure for a large region.

\section{OPERATION ENVIRONMENT}

In this section, we discuss use cases, data rate requirements, and, notably, what people in oases could follow to gain most of the possibly limited (access and) backhaul. In addition, it is discussed what could be the role and actions of other stakeholders such as governance and operators.

\subsection{Use Cases}

The simplest use case is often remote working in a place that is located at the cell edge with limited service. This use case is included in Figure 1, close to the internet access point. In this case, it is sufficient to obtain a directional antenna and a modem and to share connectivity locally using Wi-Fi. As such, the existing technology could be used. Different nations also have their broadband strategies that include plans to provide fiber or some other point of presence $(\mathrm{PoP})$ close to living and working places. Those PoPs can be exploited to build the last-mile connectivity to end users.

A more severe case is when the oasis is outside the cell, requiring a hop (or a few) to connect. The separating terrain could be hilly or forested, include rivers, have bad roads, and suffer from floods and landslides. Naturally, these are not insurmountable obstacles if the building decision has been made. A more severe limitation is missing or unreliable power grid, meaning that relays and access points have to rely wholly or mostly on renewable power sources or diesel generators, which in the future are (hopefully) run by sustainable biofuels. However, generator refueling may form a significant part of operational costs, which should be minimized (Handforth, 2019). Luckily, many areas have yearround sunlight, and it is theoretically possible to rely on renewable sources if power consumption is kept at acceptable levels. Occasionally, other renewable sources might also be available, such as wind and hydroelectric power. However, some areas do not have this privilege, and sunlight is too weak or not available in all months of the year; thus, generators or other alternative power sources are required. This is the case in the north (and very south), where global warming causes additional challenges such as permafrost melting that causes "landslide" that may damage infrastructure. In addition, snowing and icing increase challenges in those regions. The powering problem also means that sustainable battery technology should be available.

A microwave link chain may not serve just one oasis, but there could be a chain of oases that have to be served, or the link is branched at some relay or access station. As such, the early parts of the chain have to serve aggregated traffic. In addition, one relay station may have to serve several links, and one access point serves several links in addition to the local oasis.
Another deployment issue is that the number of houses and population in an oasis varies significantly. In order to give some insight, we use India as an example. The village size in India varies from less than 100 residents to more than 10,000 residents. The village of Musapura in Madhya Pradesh, India, has about 40 mud-houses with approximately 200 people. Such populations exist in small clusters (villages), which are separated from one another by a few kilometers. In some cases, however, these could be located far away from the cities. In other places, the problem could be individual houses outside the villages and far away from the main infrastructure.

We also consider a use case of smart farming using upcoming technologies such as the Internet of Things (IoT), artificial intelligence (AI), and drones for sensing, analyzing, and herd tracking, as an example, to increase production efficiency, by maximizing the use of resources and minimizing the environmental impact. Communication systems should be able to scale according to farm sizes that vary across different countries. According to Dryancour (2017), the average farm size in Australia is about 800 ha, which is 4.5 times bigger than the farm size in the United States ( $175 \mathrm{ha})$. In the European Union (EU) area, the average farm size is $17 \mathrm{ha}$, which is 47 times smaller than that in Australia.

Once the connectivity is improved, it is expected that people will use services similar to what the connected areas use currently. However, catering to the most demanding applications such as online gaming, video and TV streaming, and virtual reality is still a challenge. The current requirements of these applications are shown in Table 1, which illustrates what individual data rates might be needed. Virtual- and augmented-reality applications will find their place not only in the entertainment sector but also in healthcare, business, science, and education as discussed in Hexa-X (2021). Remote business use cases have data rate requirements similar to normal daily-living use cases, but they have higher requirements for availability and reliability. All these services need good network throughput. However, later, we shall discuss what can be done if the throughput is not sufficient for these services. Luckily, many services are readily adaptive and can change their quality level depending on connectivity. However, simultaneous usage will form aggregated traffic that increases throughput demands.

At the moment, the downlink direction is considered more dominant, and thus, the downlink usually has more capacity. However, future 6G services also include video communication (e.g., in school works, e-health, and haptics applications), in which the uplink direction also becomes more important. Moreover, situation awareness of emergency authorities may require a more dominant uplink. This means that both radio access network (RAN) and backhaul must be flexible and able to dynamically adjust the load in each direction. For example, the minimum requirements of Federal Communications Commission (FCC) in its Auction 904: Rural Digital Opportunity Fund $^{3}$ were 25/3 Mbps for downlink/uplink, whereas the baseline was set to $50 / 5 \mathrm{Mbps}$, while the data

${ }^{3}$ https://www.fcc.gov/auction/904/factsheet\#technology 
TABLE 1 | Example data rate requirements, where B denotes bytes and HD high density. Note that GB/h corresponds, on average, to a 2.2 Mbps data rate. Latency, which is an issue in online gaming, is not addressed herein. Information in the table is from online sources. ${ }^{a, b, c, d, e, f}$

\begin{tabular}{|c|c|c|}
\hline Service & Data [GB/h] & Recommended min data rate [Mbps] \\
\hline \multicolumn{3}{|l|}{ Streaming } \\
\hline Standard TV show or movie & up to 1 & 3 \\
\hline Full HD & up to 3 & 5 \\
\hline $4 \mathrm{~K}$ ultra HD & up to 7 & 25 \\
\hline \multicolumn{3}{|l|}{ Virtual reality } \\
\hline 4K CG video & & 50 \\
\hline 8K CG video & & 200 \\
\hline \multicolumn{3}{|l|}{ Video calls } \\
\hline Standard two-way & & 1 \\
\hline HD two-way & & 3.6 \\
\hline \multicolumn{3}{|l|}{ Gaming } \\
\hline Consoles & & 5/3 (down/up) \\
\hline Games & typ. about $0.1, \max 0.7$ & $5 / 3$ \\
\hline First-person shooter & & $30 / 1$ \\
\hline
\end{tabular}

${ }^{a}$ https://www. digitaltrends.com/movies/how-much-data-does-netflix-use

${ }^{b}$ https://www.zen.co.uk/blog/posts/zen-blog/2019/04/18/the-minimum-internet-speeds-for-streaming-video-in-2019

chttps://www.highspeedinternet.com/resources/how-much-speed-do-i-need-for-online-gaming

${ }^{d}$ https://www.maketecheasier.com/how-much-bandwidth-does-video-calling-use

${ }^{e}$ https://www.allconnect.com/blog/recommended-speeds-by-gaming-type

${ }^{f}$ https://www.gsma.com/futurenetworks/wiki/cloud-ar-vr-whitepaper

access requirement was $250 \mathrm{~GB}$ monthly. At the EU level, the target download speed has been $100 \mathrm{Mbps}$ for every household, but that has been recently increased to $1 \mathrm{Gbps} .{ }^{4}$ Another aspect is the data plan or data hosting contract that can be limited or unlimited, and in the former, the cost (per MB) increases if the limit is exceeded.

\subsection{Oasis}

The connectivity solutions in oases must be affordable; hence, most probably, they have to be based on widely available technology such as Wi-Fi. "All the Gs" mobile systems may not be affordable and low power, while devices offering only limited services (e.g., voice and SMS) are not sufficient. Therefore, a significant question is if $6 \mathrm{G}$ could be affordable and offer something better than $\mathrm{Wi}$-Fi, e.g., in terms of battery recharge period. However, there are many other aspects affecting energy consumption than wireless connectivity (e.g., air conditioning).

In general, it is either local capacity or backhaul capacity that forms the limit. Base stations are capacity limited, and thus, problems may occur once the number of simultaneous users increases, and because of that, the system has to be planned accordingly. Since backhaul may remain a bottleneck in a sense that it does not offer "unlimited" services, people have to learn how to make the best use of the limited connectivity. In a selfish behavior, each individual or household streams and plays the games that they want, and the result may be insufficient quality for all. That is what we have done in the past in urban areas (cities), and the user experiences are compromised. Therefore, people should act differently. For example, they could agree to watch the same stream or broadcast at the same time. However,

${ }^{4}$ https://ec.europa.eu/digital-single-market/en/broadband-strategy-policy this requires broadcast capabilities for the oasis and then multicasting to different devices, which are yet to become a reality. It would require streaming companies to permit such usage scenarios, and the billing would need to be managed accordingly. The oasis could include edge computing resources in caching the contents temporarily, and this could be utilized in such situations, but how this could be done still needs to be solved. In addition, since the backhaul might carry aggregated oasis traffic, cooperation between oases might be required. If sufficient advances are made in $6 \mathrm{G}$ research, the oasis together with the backhaul network can evolve to be capable of selforganizing and self-coordinating for better load balancing along the end-to-end communication path (3GPP, 2020).

In $6 \mathrm{G}$, we envision that people can also prioritize certain applications, at least at certain times of the day. For example, e-education, e-health, and filling and digging of government forms and helpful data could be given a higher priority during daytime and thus priority access before leisure web browsing and streaming. E-education and e-health may include the use of twoway video, which makes them rather challenging applications, and thus, limited backhaul has to be used wisely. We can further reduce the demand on the backhaul network and henceforth avoid congestion by pre-fetching the content. The content (including government forms/educational content) can also be stored locally on the servers. The filled-in forms, evaluation sheets, assignments, etc. can be transferred out from the local server to the central servers. Emergency and safety critical services may also, if they are needed, reserve a part of the capacity, causing similar limitations. However, the authorities need to ponder whether this cause should be communicated to the users to inform them of the reason for limited services so that people would be more aware. Such smart use of resources calls for a new kind of thinking and cooperation between many actors and 
disciplines. Different cultural aspects have to be taken into account as well. Recent and expected advances in distributed learning (e.g., federated learning) can help collaboratively train a shared model through the local data, and thus, only the upload model updates instead of raw data to centralized servers to further alleviate the burden on the backhaul link. Along with caching, such local learning could be performed on cheap commercial offthe-shelf (COTS) hardware.

\subsection{Other Stakeholders}

In many places, authorities such as police, ambulance, and fire department units (public safety in general) suffer from limited connectivity and the lack of modern services such as sending and receiving large files and images or even two-way video to increase situation awareness. These would urgently be needed in various disasters such as flooding, landslides, and forest fires but also to alert people. The recent trend is to apply the civilian, commercial systems and infrastructure to reduce costs. With the same reasoning, it might be possible that as an exchange for government financial support for improving connectivity in rural and remote areas, these systems could be used for providing connectivity to authorities. However, instead of the fixed share, this should be done on a need basis to release as much of the (limited) backhaul capacity as possible for regular usage. It could be organized through an "on-demand" authorities' slice.

Governance is also responsible for frequency regulation that is of interest where wireless terrestrial backhaul is considered. Often, frequencies are allocated statically to a region or nation, but this has resulted in inefficient usage of this scarce natural resource with high spectrum fees. Instead, a flexible frequency allocation would be more efficient. This is especially true in rural and remote areas where interference is of less concern since, many times, the allocated frequencies are unused in these areas. Consequently, therein, a larger bunch of suitable spectrum might be allowed for efficient wireless backhauling. This could mean changes to the rules of the existing frequency allocations. It could also be managed on a voluntary basis, if frequency owners agree to cooperate. In any case, inefficient frequency utilization benefits no one. Flexible spectrum usage could also ease problems that are encountered when local access is provided in larger areas such as big farms, mines, and other industrial sites. For example, TV white spaces could be used for this purpose if accurate spectrum awareness is ensured (Kumar et al., 2016).

Local access could be offered by operators or local service providers or community, in which case, these could be micro operators and in some cases based on the principles of nonprofit. Local access could also be private networks, e.g., in farms and mines. In any case, they need backhaul services that could be managed by others. National roaming should be allowed to make the small operator concept viable since people have to move around, from a small operator's area to a large operator's area and vice versa (Chaoub et al., 2021).

It has been argued that there should be basic service (i.e., certain data rate) that is either of low cost or even free. In addition, premium service may be offered. If the basic service is free, this concept is called a freemium service (Handforth, 2019). It is important to describe the rules of this concept: (1) what the data rate is in the basic service (especially if public funding/ regulation is used to push solutions), (2) how it evolves with time, (3) when the premium service is possible, (4) whether it is possible to provide it at the cost of the basic service, and so on. All these are the concerns in the local access and the backhaul service, indicating that these are tightly coupled. This calls for new business models to be developed as a part of the $6 \mathrm{G}$ research and standardization activities, which would be certainly different than the existing models. This would also require training of the operators and technical support (or volunteers) at the local site. Due to the very nature of differentiated services and need-based services design, we would need enhanced and AI-enabled network planning tools to be developed.

\section{BACKHAUL}

Microwave links or self-backhauling (e.g., IAB) links are typically set to be line-of-sight (LoS) links with highly directional antennas. This means that the received signal experiences more or less a single-path propagation. Depending on antenna heights and obstacles en route, the path loss often follows the free-space model until some point where the Fresnel zone starts closing. If the antennas are low (as it may be if costs are a factor) or the terrain is hillier, then additional path loss has to be included. At higher frequencies, mist, rain, and snow (wet or dry) cause additional losses $(\mathrm{dB} / \mathrm{km})$ that have to be handled, e.g., using a power margin, using power control by increasing power in bad conditions, or using adaptive modulation and coding (MODCOD), i.e., high MODCOD for good channel conditions and low MODCOD for bad channel conditions. Power saving is also a factor to be considered in order to avoid excess power usage.

Multicarrier orthogonal frequency-division multiplexing (OFDM) technology is the de facto method nowadays. However, it results in inefficient power amplifiers (PAs), up to $35 \%$ efficiency, with modern design and typical $7 \mathrm{~dB}$ back-off as shown by Singya et al. (2017) and Nikandish et al. (2020) (only $15 \%$ efficiency in some cases). Single-carrier technology and especially the constant envelope Gaussian minimum-shift keying (GMSK) modulation (used in GSM) is also a pragmatic solution since it would allow PAs that have about $60 \%$ efficiency, thus reducing power consumption. Its drawback is thought to be the demanding equalizer. On single-path channels, however, there is no need for computationally demanding, multi-tap, and long-delay line equalizers since a single-tap equalizer is sufficient. The downside is, however, that constant envelope GMSK supports only quadrature phase-shift keying (QPSK) modulation. It can be argued that since future radios are software based, they could include both GMSK and OFDM modes where the latter is used when power saving is not critical and higher data rates could be used.

Another point to analyze is the used modulation and coding scheme. Typically, the QPSK modulation and a half-rate (or lower) coding is used to provide basic, robust service, whereas higher modulation orders (up to 4096QAM or even higher) and slight coding are used for higher data rates. Table 2 includes the 
TABLE 2 | Required SNR per symbol for different modulations (without coding) when the target BER is $10^{-5}$, representative coding rates for each modulation and data rates relative to QPSK.

\begin{tabular}{|c|c|c|c|c|}
\hline Modulation & SNR/symbol [dB] & Additional SNR wrt QPSK [dB] & Coding rate & Data rate wrt QPSK $[\times]$ \\
\hline QPSK & 12.5 & 0 & $1 / 2$ & 1 \\
\hline 16QAM & 19.5 & 7 & $3 / 4$ & 3 \\
\hline 64QAM & 25.8 & 13 & $5 / 6$ & 5 \\
\hline 256QAM & 31.5 & 19 & $5 / 6$ & 5.7 \\
\hline 1024QAM & 37.5 & 25 & $5 / 6$ & 8.3 \\
\hline
\end{tabular}

required signal-to-noise ratio (SNR) per symbol for different modulation schemes without coding. Usually, light coding is used such that the coding gain is not that much, but a few $\mathrm{dB}$ could be counted in some cases. Nevertheless, representative coding rates and related data rates corresponding to the QPSK modulation are specified. The coding is included in data rate evaluations, but reference and control symbols, synchronization symbols, etc. are ignored. For a target bit error rate (BER) of $10^{-5}$, it can be concluded that from the power usage perspective, it is better to use more bandwidth and low-order modulation than narrow bandwidth and high-order modulation (compare required SNR and relative data rate columns). However, in reality, bandwidth availability might be a concern. In rural and remote areas, largerspectrum chunks can be made available, at least regionally, if it is possible to use frequencies flexibly. Therefore, frequency regulation has an important role in energy-efficient rural and remote area wireless backhaul solutions.

The required received power (SNR per symbol) can be turned into sensitivity. For example, the QPSK mode of RAy 3 link shown in Racom (2021) has a sensitivity level of $-85 \mathrm{dBm}$, and 64QAM has $-70 \mathrm{dBm}$ sensitivity with $56 \mathrm{MHz}$ bandwidth. This $15 \mathrm{~dB}$ sensitivity difference is in line with the values in Table 2. The noise power at $56 \mathrm{MHz}$ bandwidth is $-96 \mathrm{dBm}$ such that the $-85 \mathrm{dBm}$ claimed sensitivity includes realistic noise figure and required SNR with coding. If the other modulations in RAy 3 link are considered and compared with QPSK, it seems that, in practice, the difference in additional SNR is somewhat larger than that in Table 2. A comprehensive study on various existing modulation schemes and their comparison with futuristic energyefficient modulation schemes such as star-QAM and hexagonalQAM are detailed by Singya et al. (2021).

The main aim of this section is to analyze the required power at a relay station and local area access point that includes both the link and access. These are evaluated with respect to the current state of the art and the capability to rely on solar power with a reasonable panel size. It will indicate the innovation potential for $6 \mathrm{G}$.

\subsection{Minimum Required Transmitted Power}

Since it is impossible to give exact values for all the situations, we provide results using some practical frequencies, antenna heights, and antenna gains representing typical values found in the literature review when lightweight microwave relays are considered. The best-case channel model assumes that diffraction occurs due to the Earth's curvature when $60 \%$ of the first Fresnel zone is no longer open, which is usually seen as a

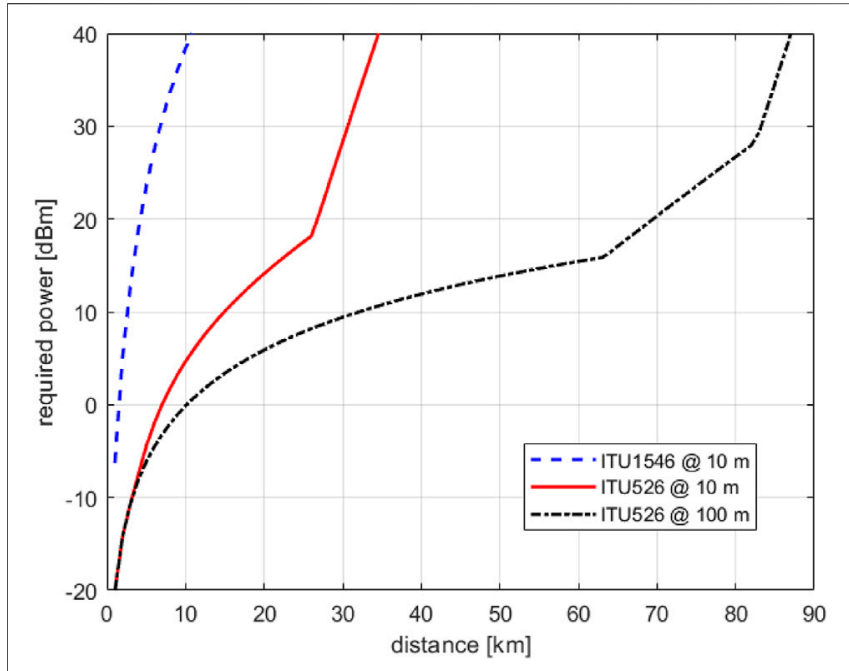

FIGURE 2 | Required PA power at $700 \mathrm{MHz}$ carrier frequency as a function of link distance with $40 \mathrm{MHz}$ bandwidth and QPSK modulation with 1/2-rate coding.

rule for free-space propagation. This is included in the ITU-R P: 526-13 propagation model explained in ITU-R (2013b). For environments with more obstacles, such as hilly regions, models such as ITU-R P:1546-5 detailed in ITU-R (2013a) are used although it has been observed to be too pessimistic in many cases as discussed by Omslandseter et al. (2018). In both models, we have modified them so that after the radio horizon, the additional path loss is $2 \mathrm{~dB} / \mathrm{km}$, though opinions about this value vary in the literature. However, this helps to observe the radio horizon in the figures.

The carrier frequencies considered are $700 \mathrm{MHz}, 2.5 \mathrm{GHz}$, and $5 \mathrm{GHz}$, and the antenna heights considered are 10 and $100 \mathrm{~m}$, where the first one represents a lightweight case where all antennas are relatively low and the last one represents a better case where antennas could be on the mountain tops or in high towers (e.g., existing infrastructure such as TV towers could be utilized). At the local site, the antenna height also affects the provided coverage area (since the link and local access are typically at the same mast). The antenna gains are $13 \mathrm{dBi}$ (Yagi) for $700 \mathrm{MHz}$ and 21 and $26 \mathrm{dBi}$ for the higher frequencies, corresponding to about $60 \mathrm{~cm}$ antenna disk. The results are for QPSK modulation and $40 \mathrm{MHz}$ bandwidth. If more (or less) bandwidth is used, the values have to be scaled accordingly. In addition, if higher MODCOD is used, the 


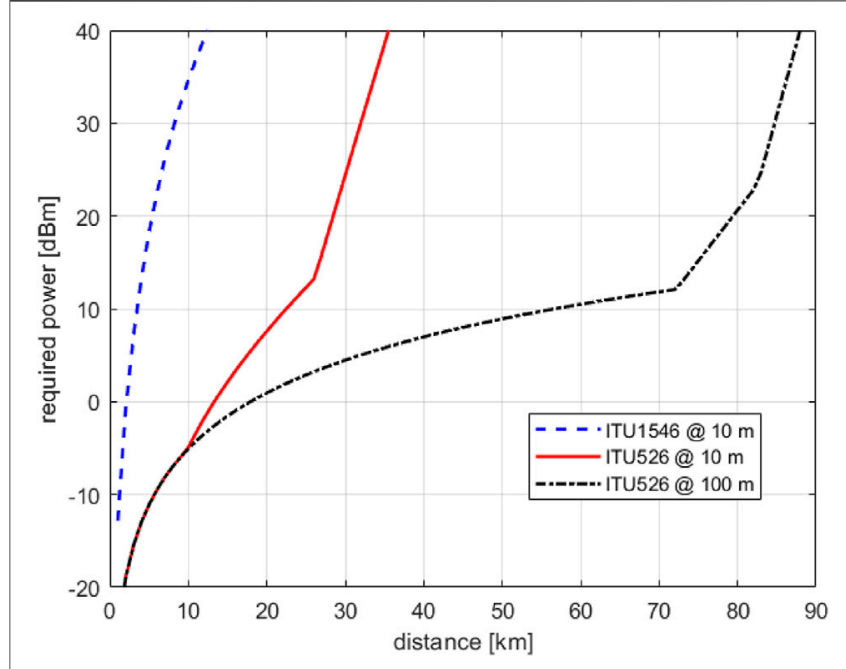

FIGURE 3 | Required PA power at $2.5 \mathrm{GHz}$ carrier frequency as a function of link distance with $40 \mathrm{MHz}$ bandwidth and QPSK modulation with $1 / 2$ rate coding.

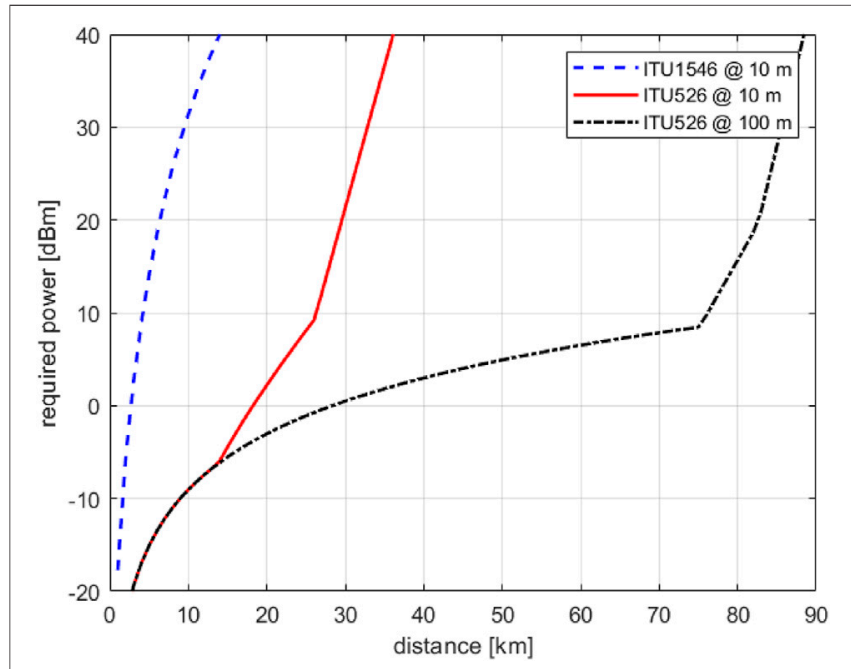

FIGURE 4 | Required PA power at $5 \mathrm{GHz}$ carrier frequency as a function of link distance with $40 \mathrm{MHz}$ bandwidth and QPSK modulation with 1/2-rate coding.

required extra power can be checked from Table 2 . One has to note that the low-end values do not include a power margin for bad weather needed at high frequencies. Moreover, cable losses are insignificant if the RF part is close to the antenna as assumed herein.

The thermal noise density of $-174 \mathrm{dBm} / \mathrm{Hz}$ and noise figure in base stations could be rather low as shown in Huawei (2019). Hence, we use $2 \mathrm{~dB}$ as a reference. Figures $\mathbf{2}-\mathbf{4}$ show the PA output power at various frequencies for different link distances. It can be observed that at $100 \mathrm{~m}$ antenna heights, the ITU526 follows the free-space path loss long before the diffraction occurs, and the radio horizon comes even later. However, at $10 \mathrm{~m}$ antenna heights, the diffraction occurs at a much shorter
TABLE 3 | Required PA power for selected modulations at 10 and $20 \mathrm{~km}$ distances with $10 \mathrm{~m}$ antenna heights and $40 \mathrm{MHz}$ bandwidth.

\begin{tabular}{|c|c|c|c|c|c|c|}
\hline \multirow[t]{3}{*}{ Modulation } & \multicolumn{6}{|c|}{ Required PA power $[\mathrm{dBm}]$} \\
\hline & \multicolumn{2}{|c|}{$700 \mathrm{MHz}$} & \multicolumn{2}{|c|}{$2.5 \mathrm{GHz}$} & \multicolumn{2}{|c|}{$5 \mathrm{GHz}$} \\
\hline & $10 \mathrm{~km}$ & 20 km & 10 km & $20 \mathrm{~km}$ & $10 \mathrm{~km}$ & $20 \mathrm{~km}$ \\
\hline QPSK & 5 & 15 & -5 & 8 & -9 & 3 \\
\hline 64QAM & 18 & 28 & 8 & 21 & 2 & 16 \\
\hline 1024QAM & 30 & 40 & 20 & 33 & 16 & 28 \\
\hline
\end{tabular}

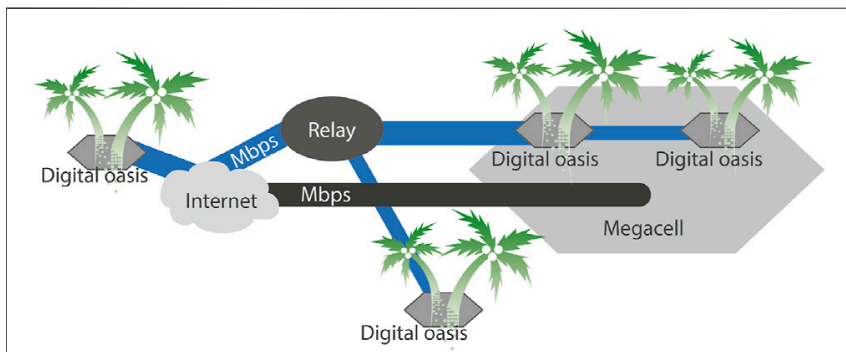

FIGURE 5 | Backhaul chain options in different cases. Note that the mega-cell needs just one backhaul.

distance, and the radio horizon is about $30 \mathrm{~km}$. If there are more obstacles (badly placed antennas), then even $20 \mathrm{~dB}$ or more additional power may be required.

Table 3 shows the required power for 10 and $20 \mathrm{~km}$ link distances using QPSK, 64QAM, and 1024QAM based on the ITU526 attenuation model and $10 \mathrm{~m}$ antenna heights. Somewhat surprisingly, the lowest frequency is not necessarily the best. The reasons are the difference on reasonable antenna gains and the fact that the diffraction point is shorter at low frequencies. Therefore, careful joint frequency and antenna selection and antenna positioning are needed. The results show that ideally rather low average power levels such as 10 or $100 \mathrm{~mW}$ are needed for 10 and $20 \mathrm{~km}$ links for QPSK and 64QAM signals. Since one link requires both directions, the power need is doubled. In addition, some other factors also affect the total power consumption as discussed in the next subsection.

\subsection{Power Consumption in Relay/Base Stations}

In the simplest case, in the middle of a backhaul chain, a tower has 2 two-way links: one for the internet access point and one for the oasis. However, towers may have more two-way links to different oases, and the link to the internet must carry aggregated traffic as illustrated in Figure 5.

At relay stations, power consumption can be modeled as (Monti et al., 2012)

$$
P_{r}(C)=P_{r, \mathrm{ag}}(C)+P_{r, \mathrm{sw}}\left(A_{r}, C\right),
$$

where $C$ represents the total aggregated capacity that the backhaul node $r$ has to relay and $A_{r}$ represents the number of microwave antennas (i.e., the number of microwave links) used at that relay, which denotes 
also the number of oases served by this relay node. $P_{r, \mathrm{ag}}(C)$ is the power consumption for transmitting and receiving the aggregate backhaul traffic. $P_{r, \mathrm{sw}}\left(A_{r} C\right)$ is the power consumption of the switch that needs to be used at any relay site to serve its neighboring backhaul links. These variables are defined as

$$
P_{r, \mathrm{ag}}(C)= \begin{cases}P_{\mathrm{L}} & \text { if } C \leq C_{\mathrm{th}} \\ P_{\mathrm{H}} & \text { otherwise }\end{cases}
$$

and

$$
P_{r, \mathrm{sw}}\left(A_{r}, C\right)= \begin{cases}0 & \text { if } A_{r}=1 \\ P_{\mathrm{sw}} \times\left\lceil\frac{C}{C_{\mathrm{max}}^{\mathrm{sw}}}\right\rceil & \text { otherwise. }\end{cases}
$$

$P_{\mathrm{L}}$ and $P_{\mathrm{H}}$ are the power consumption of the microwave antennas associated with the low and high traffic conditions, respectively. A threshold capacity $C_{\text {th }}$ is defined to classify these two traffic conditions, but also more capacity regions could be used. $C_{\max }^{\mathrm{sw}}$ is the maximum capacity of the switch, and $P_{\mathrm{sw}}$ is the fixed power consumed by the switch.

The base station power consumption comprises two categories, as discussed by Deruyck et al. (2014). The first category consists of the components whose power consumption is not load dependent, such as the rectifier, the microwave link, and the air conditioning. The second category is the equipment that has load-dependent power consumption, such as the PA, the transceiver (TRX), and the digital signal processing (DSP). The power consumption model is

$$
\begin{aligned}
P_{b s}= & n_{\mathrm{SC}} \cdot\left(f \cdot\left(n_{\mathrm{TX}} \cdot\left(P_{b s, \mathrm{FD}}+P_{b s, \mathrm{PA}}+P_{b s, \mathrm{RF}}\right)+P_{b s, \mathrm{BB}}\right)+P_{b s, \mathrm{RC}}\right) \\
& +P_{b s, \mathrm{CL}}+P_{b s, \mathrm{BL}}
\end{aligned}
$$

where $f$ is the load factor representing the number of active users. $P_{b s, \mathrm{FD}}$ denotes feeder losses, and $P_{b s, \mathrm{PA}}, P_{b s, \mathrm{RF}}, P_{b s, \mathrm{BB}}, P_{b s, \mathrm{RC}}, P_{b s, \mathrm{CL}}$, and $P_{b s, \mathrm{BL}}$ represent the power consumption of the PA, RF TRX, baseband engine, rectifier, air cooling, and microwave link (if it exists), respectively. Finally, $n_{\mathrm{SC}}$ and $n_{\mathrm{TX}}$ denote the number of sectors and transmitting antennas, respectively.

Since the antennas are usually located outside the base station site, feeder system losses need to be considered. However, these losses can be neglected and omitted if the PAs and the transmitting antennas are installed together in the same location as a part of the so-called remote radio head (RRH), or in the case of smaller and lightweight base stations. It should be noted that PAs and TRXs are deployed per transmit antenna, whereas DSP units and rectifiers are deployed per sector. Air conditioning and the microwave link are common to all sectors and serve the whole site. For further details, see for example Auer et al. (2011).

\subsection{Power Gap Discussion}

It is well known that the high-end base stations (and relays) are power hungry. For example, fully armed 5G base stations could consume $^{5} 11 \mathrm{~kW}$, which is too much for a system relying on

${ }^{5}$ https://www.fiercewireless.com/tech/5g-base-stations-use-a-lot-more-energy-than4g-base-stations-says-mtn renewable energy. Consequently, simplified relay and base stations should be used. So far, it has been shown in the previous sections that rather low power might provide sufficient data rates. Furthermore, it has been shown that some existing systems are using quite low power, but not optimally low. In this section, we discuss potential ways to improve power efficiency in $6 \mathrm{G}$.

In what follows, we provide the analysis of power needs based on energy consumption in PA depending on modulation and coding, the suitability of reasonable solar energy systems to power such connectivity, and an example discussion about mega-cells compared to usual cells to cover a remote/rural area. The difference between a mega-cell and a microwave backhaul chain is illustrated in Figure 5, whereas power-consuming parts in relay and access towers are depicted in Figure 6.

\subsubsection{Modulation and Coding Selection}

PA is probably the most power-hungry part of a relay or base station as described by Auer et al. (2011). If the efficiency is denoted by $\eta$, the consumed power is approximately

$$
P_{b s, \mathrm{PA}}=P_{a v e, \mathrm{PA}} / \eta,
$$

where $P_{\text {ave, } \mathrm{PA}}$ denotes the average output power. There are two different commonly used efficiency definitions, but we shall skip those details for now. A review presented by Vasjanov and Barzdenas (2020) shows that certain PA designs could allow a tad over $40 \%$ efficiency even with back-off but for rather narrowband signals, below $10 \mathrm{MHz}$ bandwidth. Larger bandwidths are usually achieved with more traditional design, where the efficiency is between $5 \%$ and $30 \%$ as seen from Vasjanov and Barzdenas (2020, Table 6).

If $35 \%$ and $60 \% \mathrm{PA}$ efficiency are compared, the latter requires 1.4 times less power $(1.4 \mathrm{~dB})$. However, if the average efficiency was $15 \%$, the difference would be four times (or $6 \mathrm{~dB}$ ). Therefore, the low-efficiency PAs should be avoided in remote area backhaul systems relying on solar energy or targeting at savings on refueling the generator. The back-off also has other consequences. For instance, if the average power is $X \mathrm{dBm}$ and back-off $Y \mathrm{~dB}$, the maximum power that has to be provided for the PA is $X+Y \mathrm{dBm}$ such that, e.g., instead of $40 \mathrm{dBm}$ average power, the system has to be able to offer $46 \mathrm{dBm}$ peak power if $6 \mathrm{~dB}$ back-off is assumed. The OFDM back-off can be reduced using clipping and other techniques. In the extreme case, $2 \mathrm{~dB}$ or even lower back-off levels can be achieved but with excessive usage of reference symbols as shown in Saarnisaari and de Lima (2020).

In this light, the constant envelope signals have $6 \mathrm{~dB}$ (usual back-off) $+2-6 \mathrm{~dB}$ (PA efficiency difference) or a total of 8-12 dB power advantage over the OFDM or single-carrier QAM signals. Furthermore, techniques and technology to save power in both relays and access points could be in the $6 \mathrm{G}$ agenda. The use of learning methods of selecting power-saving parameters (transmit power level, bandwidth, MODCOD, active elements, and sleep/ wake periods) in the overall system is an interesting topic that can be enhanced in the future $6 \mathrm{G}$ system. This includes predicting temporal data rate and usage models with flexibility to react to unexpected demands as discussed by Saarnisaari (2014). Since the 


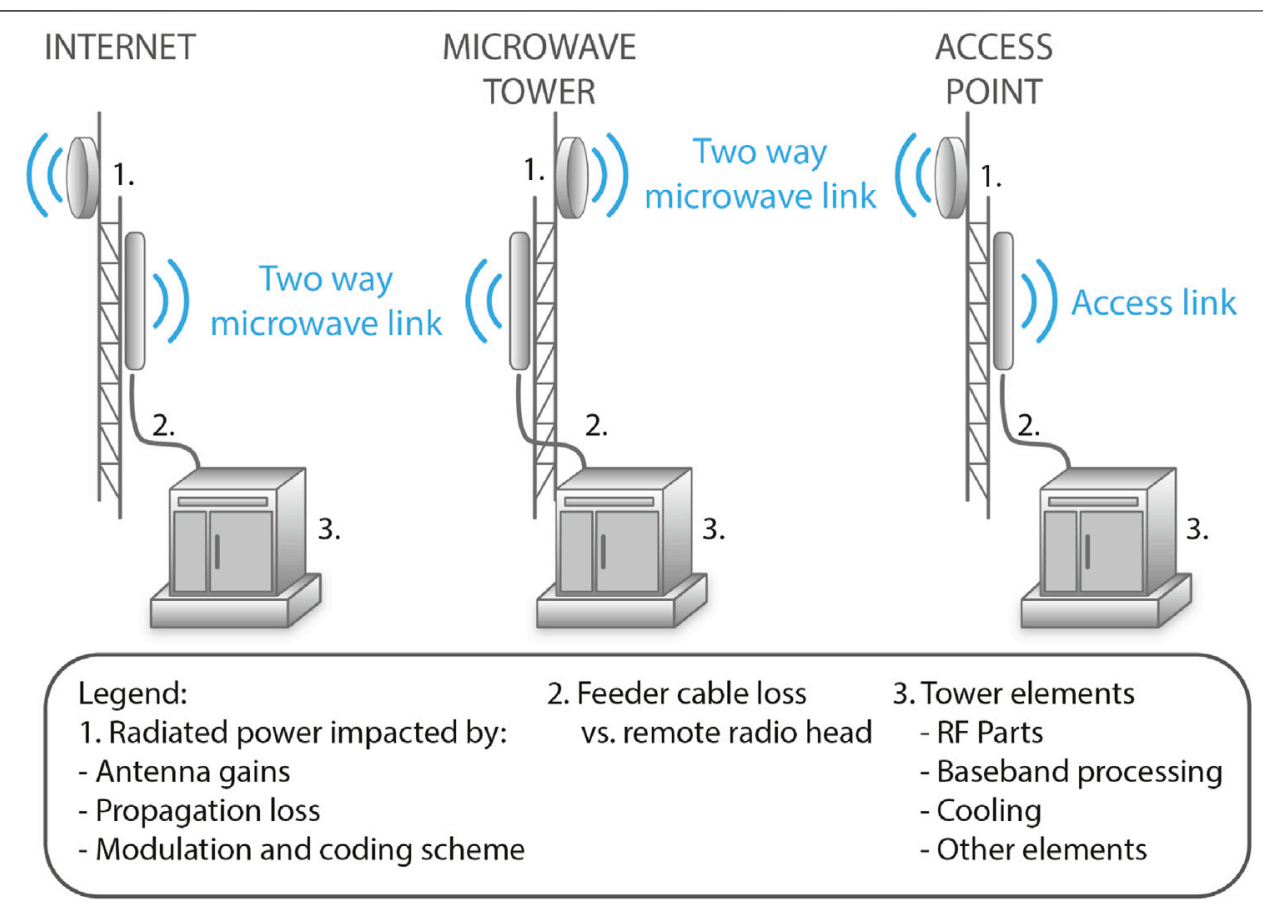

FIGURE 6 | Power consumption points in a backhaul chain connected to an access point.

trend is towards fully virtual signal forming, the signal library could include both constant envelope and high data rate (e.g., OFDM) waveforms in the future. Another questions is how economical it is to standardize and implement several signal waveforms, especially if they are highly complex and flexible, since their interoperability may become an issue.

There is also room for developing low-power beamforming hardware and software in 6G. This is an important aspect since beamforming allows us to increase data rates or range and adjust radiation patterns based on need. If the base station operates through analog beamforming, only one PA is required as well as just one digital-to-analog converter (DAC), which is the most power-hungry hardware block in the transmitter. In the digital beamforming, all antennas are with a PA and DAC. However, PAs would have lower power requirements than in the analog case. Hybrid designs are also possible. The interplay between PA, hardware imperfections, and modulation schemes for practical deployment also needs to be explored in the future as highlighted by Shaik et al. (2019).

\subsubsection{Is Solar Power Enough?}

If the numerical values in Auer et al. (2011, Table 1) are observed, it can be concluded that the average PA power forms a half or a quarter of the total power consumption of one TRX chain. The lower share occurs with low TRX powers since other parts tend to consume a fixed amount. We conservatively use a quarter in our example calculations. Furthermore, we assume RRH are installed such that feeding losses can be neglected. We consider $31 \%$ PA efficiency so that if a constant envelope modulation is used with $60 \%$ efficiency, then $3 \mathrm{~dB}$ less power is needed than shown in the following evaluation. The starting point is Table 3. The PA efficiency increases the required power by $5 \mathrm{~dB}$ and
TABLE 4 | Total power consumption per TRX chain for selected modulations at 10 and $20 \mathrm{~km}$ distances with $10 \mathrm{~m}$ antenna heights.

\begin{tabular}{|c|c|c|c|c|c|c|}
\hline \multirow{3}{*}{ Modulation } & \multicolumn{6}{|c|}{ Consumed power $[\mathrm{dBm}]$} \\
\hline & \multicolumn{2}{|c|}{$700 \mathrm{MHz}$} & \multicolumn{2}{|c|}{$2.5 \mathrm{GHz}$} & \multicolumn{2}{|c|}{$5 \mathrm{GHz}$} \\
\hline & $10 \mathrm{~km}$ & $20 \mathrm{~km}$ & $10 \mathrm{~km}$ & $20 \mathrm{~km}$ & $10 \mathrm{~km}$ & $20 \mathrm{~km}$ \\
\hline QPSK & 16 & 26 & 6 & 19 & 2 & 14 \\
\hline 64QAM & 29 & 39 & 19 & 32 & 13 & 27 \\
\hline 1024QAM & 41 & 51 & 31 & 44 & 27 & 39 \\
\hline
\end{tabular}

other consumption by $6 \mathrm{~dB}$, such that in total $11 \mathrm{~dB}$ more than the average transmission power is needed. The values for the required power are indicated in Table 4.

The values in Table 4 seem to be small, below $1 \mathrm{~W}$, except for $1024 \mathrm{QAM}$ at $700 \mathrm{MHz}$, where even $100 \mathrm{~W}$ is required. These values are for the $40 \mathrm{MHz}$ bandwidth. These correspond to raw link data rates of 40,200 , and $330 \mathrm{Mbps}$ if the coding rates in Table 2 are applied. If more capacity is needed, as may happen in aggregated traffic nodes, more power is needed, e.g., for other TRX chains. Furthermore, often more than one link is required if a tower is in the middle or in the access points that have a microwave link and access TRX. This duplication means that in reality 3-6 dB (four TRX) more power is often required. It is interesting to compare these numbers with those of $\mathrm{Wi}-\mathrm{Fi}$ routers that seem to consume about $6 \mathrm{~W}$ on average and $13 \mathrm{~W}$ maximum. ${ }^{6}$ Thus, much can be done to improve the energy efficiency.

$\overline{{ }^{6} \mathrm{http}: / / \text { www.tpcdb.com/list.php?type }}=11$ 
These required total power values should be compared with what reasonable solar panel systems can produce and store for operating all year round, $24 \mathrm{~h}$ a day, 7 days a week. Furthermore, since the Sun does not shine $24 \mathrm{~h}$ a day, the energy must be stored (into batteries) for night-time use. Energy transformation losses should be accounted for. Finally, it is often easier to consider daily consumption in $\mathrm{kWh}$. Consequently, the power levels 20,30, and $40 \mathrm{dBm}$ mean 2.4, 24, and $240 \mathrm{Wh}$ /day, respectively, if used constantly. A standard solar panel consists of 60 solar cells and is about $1.6 \mathrm{~m}^{2}$, though larger panels with more (and smaller) cells are developed. The output power depends on the quality of cells and the panel. A tendency is that these are improving, and the efficiency of the panel has increased from $15 \%$ up to $22 \%$, or peak power has risen from 240 to $350 \mathrm{~W}$ (in the standard panel) or even higher. ${ }^{7}$ The average daily production depends on available radiation, which varies depending on the location and conditions. Notably, there could be substantial location-based monthly variation on the power production. As an example, a $260 \mathrm{~W}$ standard panel produces $600 \mathrm{Wh} /$ day in southern Finland, on average, ${ }^{8}$ but in the northern Finland, it produces about $10 \%$ less during sunny period, whereas during the period between November and February, production is minimal. In central Finland, the annual Sun radiation power is about $900 \mathrm{kWh} / \mathrm{m}^{2}$ and roughly $1,600-1,800 \mathrm{kWh} / \mathrm{m}^{2}$ in Lisbon or India. ${ }^{9,10}$ Therefore, it can be expected that in Lisbon or in India, one $260 \mathrm{~W}$ standard panel would produce about $1,200 \mathrm{Wh} /$ day. In fact, some of the recent energy-efficient panels are about 1.4 times higher, i.e., 840 and $1700 \mathrm{Wh} /$ day in southern Finland and Lisbon/India, respectively. In this light, high capacity can be achieved in a $10 \mathrm{~km}$ link using a single panel even though traffic is two-way. However, a more comprehensive analysis is provided below.

In this analysis, we assume 64QAM and thus $30 \mathrm{dBm}$ over a $10 \mathrm{~km}$ link. Furthermore, one access sector uses QPSK and requires $34 \mathrm{dBm}$ over a $3 \mathrm{~km}$ cell as discussed in section 4.4 . With 16QAM, $47 \mathrm{dBm}$ would be needed or 20 times more power than that for QPSK. In both cases, a $40 \mathrm{MHz}$ bandwidth and $2.5 \mathrm{GHz}$ carrier are assumed. We compare different backhaul tower and access sites. A simple tower in the middle of the chain contains two links, say to the internet site and an access site. Therefore, it has four TRX chains or requires $36 \mathrm{dBm}$. The second tower has three links, one to the internet site and two to access sites. The link to the internet site has to carry the aggregated traffic, meaning that it requires at least $3 \mathrm{~dB}$ more power than the other links. This tower has six TRX chains, and consequently, in total, it requires (at least) $41 \mathrm{dBm}$. The access sites include a backhaul link with two TRX chains, and one includes an omnidirectional cell and the other four sectors. In the latter case, the backhaul link has to serve the aggregated traffic or requires (at least) $6 \mathrm{~dB}$ more power. Consequently, the one-sector

${ }^{7}$ https://www.cleanenergyreviews.info/blog/most-efficient-solar-panels

${ }^{8}$ https://www.keravanenergia.fi/fi/energiaremppa/aurinkopaneelit/hyodyllista-tietoaaurinkopaneeleista/

${ }^{9}$ https://fi.wikipedia.org/wiki/Aurinkoenergia

${ }^{10} \mathrm{https} / / /$ en.wikipedia.org/wiki/Solar_power_in_India
TABLE 5 | The required daily power consumption for a few backhaul and access sites. The numbers in brackets are for 16QAM. The number of panels is calculated based on 1,700 Wh daily production.

\begin{tabular}{lcc}
\hline Site & Daily power consumption [Wh/day] & Number of panels \\
\hline $\begin{array}{l}\text { Backhaul } \\
2 \text { links }\end{array}$ & 100 & 1 \\
3 links & 305 & 1 \\
Access & & \\
1 link, 1 sector & $170(3,400)$ & $1(2)$ \\
1 link, 4 sectors & $680(13,600)$ & $1(8)$ \\
\hline
\end{tabular}

access site consumes $38.5 \mathrm{dBm}$ and the four-sector site $44.5 \mathrm{dBm}$. The access is the dominating power consumer in this case. The results in Table 5 indicate that a single panel is often sufficient if the required data rates remain limited and bandwidth can be flexibly increased for increasing link data rate. As a reminder, the used data rates are about $40 \mathrm{Mbps}$ for the QPSK access (total sector) and $200 \mathrm{Mbps}$ for a basic link. Furthermore, these calculations are just exemplary since they assume continuous usage and equal consumption for the receiver TRX chain and other needs are not included. However, these provide a view on different possibilities.

Since solar power is not available $24 \mathrm{~h}$ /day and year-round in some areas, solar power can be supplemented by other off-grid energy sources, such as wind energy, hydrogen cells, and others. Over the years, large wind farms have been built across the world as an alternative to solar power. As with solar power, some regions have strong winds throughout the year, whereas others may have only some windy months in a year. In studies performed at Rochester, New York (Habibzadeh et al., 2017), solar irradiation and wind speed are observed to have complementary nature and can thus be very useful in providing off-grid sustainable power. Habibzadeh et al. (2017) demonstrated that solar and wind energy have the potential to provide sufficient power for medium-powered systems (i.e., $1-10 \mathrm{~W}$ ). In the case of wind energy, the amount of harvested power was $47 \mathrm{dBm}(50 \mathrm{~W})$, and in the case of solar power, the amount of harvested power was $42 \mathrm{dBm}(15 \mathrm{~W})$. Though wind turbines have high maintenance and deployment costs, their efficiency is expected to improve considerably in the future. Finally, as a note, it is worth mentioning that the efficiency of an inverter has to be included in the power calculations.

\subsubsection{Further Possibilities}

One way to reduce the number of towers and their heights is to utilize diffraction, wherein a signal propagates beyond LoS obstacles. The problem is how this phenomenon can be efficiently utilized. Network planning and propagation estimation tools are needed for simple and cost-effective planning. Works have been carried towards this as shown in Pérez-Peña et al. (2020) and Kusuma and Boch (2021), but further progress would be required. Notably, reliable and accurate diffraction-based propagation models are needed to design such links in an easy way.

On the other hand, intelligent reflecting surfaces (IRSs) are among the latest breakthrough technologies of the $6 \mathrm{G}$ ecosystem, used to passively reflect the signal without amplification. The 
received signal power decreases proportionally to the square of the IRS area and to $\left(d_{\mathrm{TX}} \cdot d_{\mathrm{RX}}\right)^{2}$, where $d_{\mathrm{TX}}$ is the distance between the transmitter and IRS and $d_{\mathrm{RX}}$ is the IRS-to-receiver distance (Özdogan et al., 2019; Wu and Zhang, 2019). Accordingly, IRS panels can be regarded as a promising smart relay solution to overcome coverage issues in shadowed networks whenever placed close to either the transmitter or the receiver (e.g., closing coverage gaps in low-density populated areas or in difficult terrains such as hills, mountains, and jungles), while simultaneously achieving significant energy savings.

\subsection{Oasis Cell Versus Mega-Cell}

In this section, we provide example calculations to compare mega-cell and oasis cell approaches since these affect the required backhaul links. It is assumed that the radius of the mega-cell is $30 \mathrm{~km}$ and the antenna height is $100 \mathrm{~m}$, as considered in the other numerical examples. The radius of each oasis cell is $3 \mathrm{~km}$ such that the mega-cell area covers 100 oasis. If the megacell has 12 sectors, each sector has to support eight to nine oases. In a fair comparison, the mega-cell has to support the aggregated data rate of the oasis cells in its sector. This means that mega-cell sectors have to have higher data rate and longer connectivity distance.

The needed higher data rate can be covered by an increased bandwidth $(9 \mathrm{~dB}$ more power) or a higher-order modulation ( $25 \mathrm{~dB}$ more power, see Table 2 ) or by a hybrid of these two. If $2.5 \mathrm{GHz}$ is taken as the carrier frequency, $40 \mathrm{MHz}$ bandwidth with QPSK over $3 \mathrm{~km}$ distance requires at the minimum $23 \mathrm{dBm}$, whereas for $30 \mathrm{~km}$ in a mega-cell, it requires $29 \mathrm{dBm}$, calculated using the ITU526 model considering 10 and $100 \mathrm{~m}$ base station antenna heights with a $2 \mathrm{~m}$ user antenna height. The antenna gain is $21 \mathrm{dBi}$ in the mega-cell tower and $5 \mathrm{dBi}$ in the oasis cell tower, while the user has $0 \mathrm{dBi}$ antenna gain. The QPSK baseline difference is not so dramatic due to antennas. However, if the required higher data rate was counted, each sector of the megacell would need at least $38 \mathrm{dBm}$ TRX power. If all 12 sectors are counted, $48 \mathrm{dBm}$ (or nearly $100 \mathrm{~W}$ ) power is needed (assuming all sectors are active) in the mega-cell. This $2.4 \mathrm{kWh} /$ day requires a larger solar energy system. This is also the total power the megacell requires for the access, whereas the total power of oasis-cellbased access requires $20 \mathrm{~dB}$ more power since it has 100 cells. Altogether, the total power needed for access in the oasis cell system is a little smaller. If the oasis cells were sectorized, by a factor of 3-5, then 5-7 dB more power would be needed. However, individual oasis cell towers could be solar powered following the reasoning in section 4.3.2, such that each small cell TRX requires about $34 \mathrm{dBm}$ or about $50 \mathrm{Wh}$ /day.

The mega-cell might require only one backhaul link, but it has to handle all aggregated traffic, i.e., the traffic of 100 oases in the considered example. In the oasis cell deployment, the oases may be connected by a few backhaul links. If it is two, then each link has to handle $50 \%$ of the total traffic (assuming equal share), or at least $3 \mathrm{~dB}$ less power. If the distance to the area center from the internet is $40 \mathrm{~km}$, the mega-cell backhaul link is $40 \mathrm{~km}$, whereas the main oasis cell backhaul link is about $20 \mathrm{~km}$, assuming one relay tower is needed. Based on the results presented in Figure 3, it can be seen that about the same power is required (for the baseline QPSK), if the oasis cell tower cannot be in the LoS environment, in which case it would need $6 \mathrm{~dB}$ less power. Hence, the total difference is that the mega-cell backhaul requires at least twice as much power as the oasis cell main backhaul.

The oasis cell that serves as a gateway to the main backhaul link requires the most power since it has to support the main backhaul and then serve as a backhaul to one or more oases. Moreover, these links have aggregated traffic if there is a chain of oases after each continuing backhaul. Since the backhaul link (with aggregated traffic) is the dominant power consumer in these edge oases' towers, their backhaul power consumption could be close to that of the backhaul of the mega-cell and form a bottleneck for the system. If power availability is an issue when serving aggregated traffic, then the data pipe should be divided, but that means increasing the number of backhaul towers, which needs exploration of the performance-cost trade-off.

Even this simplified example shows that it is not easy to design a backhaul chain that includes tens of oases. In addition, power and data rate requirements vary depending on where the relay is located in the backhaul chain. This fact has to be taken into account when the networks are actually implemented. Furthermore, robustness in the form of multiple backhaul routes, as backups, should be considered. It can also be concluded that minimizing aggregated traffic becomes an important factor if operated in a power-limited environment. This means that smart edge caching, multicasting, and online streaming techniques will play a very important role in the future.

In a nutshell, mega-cells are not necessarily the best solution for remote and under-connected regions. It is a matter of fact that to deploy reliable mega-cells, narrower sectors are needed to increase the coverage, which means more sectors per cell (e.g., 36 in the case of Facebook's SuperCell), leading to a higher number of TRX chains.

\section{CONCLUSION AND DISCUSSIONS}

In Figure 1, we provided various challenges that have to be addressed during $6 \mathrm{G}$ development when remote area connectivity problems are considered. Here, we discuss what possible solution pathways we identified to solve some of these challenges. Together, the challenges and the solution pathways form a solid basis for further $6 \mathrm{G}$ studies that addresses the rural and remote area connectivity problems. These pathways are summarized in Figure 7. Before looking at the details, we mention that as noted in this paper, new research ideas and innovations need to emerge within the next decade to help close the digital divide. Consequently, we provide in Table 6 our views about what these technical elements could be.

It was observed that there is room for technical development in $6 \mathrm{G}$ when low-power terrestrial backhaul systems are considered for rural and remote areas, especially when affordable but sufficient solutions are sought. Indeed, power autonomy is one of the main driving forces when a power grid is not available or it is not reliable. The same goal is valid for a RAN and user equipment when future $6 \mathrm{G}$ solutions for rural 


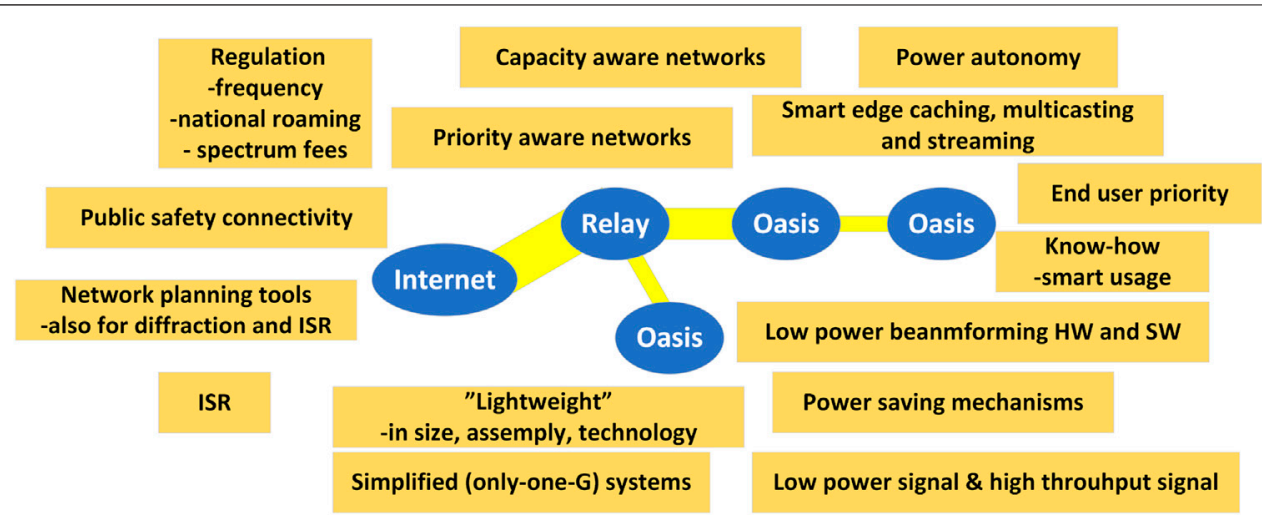

FIGURE 7 | Terrestrial wireless backhaul requirements and some solution pathways.

TABLE 6 | Evolution of terrestrial wireless backhauling in remote areas from $5 G$ to $6 G$.

\begin{tabular}{|c|c|c|}
\hline $\begin{array}{l}\text { Technology } \\
\text { elements }\end{array}$ & Current status (5G when appropriate) & Potential new features (6G) \\
\hline Spectrum & $\begin{array}{l}\text { Conventional bands (e.g., E-band), static } \\
\text { allocation }\end{array}$ & New bands (e.g., TV and Wi-Fi), flexible allocation, and eased regulation \\
\hline Signal & $\begin{array}{l}\text { Proprietary signal stacks, specialized } \\
\text { hardware }\end{array}$ & Standardized, virtualized, and/or open signal stacks, integration with COTS equipment \\
\hline Backhaul planning & LoS-based propagation models & Enhanced propagation estimation tools (e.g., diffraction based) \\
\hline Cells & Macro-cells, fixed wireless & Adaptive case-dependent cell deployment: low-power cells and/or mega-cell \\
\hline $\mathrm{IAB}$ & Dynamic resource multiplexing & Scalable resource multiplexing for progressive rural inclusion \\
\hline Cooling & Required in complex sites & Simple sites without cooling \\
\hline PA & Inefficient power amplification due to OFDM & The signal toolkit has signals suited for more efficient PAs (e.g., GMSK) \\
\hline Antennas & Analog and/or digital beamforming & $\begin{array}{l}\text { Low-power beamforming and integration with breakthrough technologies (e.g., intelligent reflective } \\
\text { surfaces) }\end{array}$ \\
\hline Traffic & Asymmetric (downlink dominant) & Balanced (uplink/downlink) for richer services \\
\hline Service plans & Basic & Basic and premium (even freemium) to reduce the digital divide \\
\hline Slicing & QoS based & Dedicated slice for remote connectivity \\
\hline Energy & $\begin{array}{l}\text { Power grid, hybrid renewable, and diesel } \\
\text { generators }\end{array}$ & Large-scale and self-sufficient renewable sources \\
\hline System optimization & Limited, selfish intelligence & $\begin{array}{l}\text { Collective intelligence, self-coordination, and self-healing based on user needs and network } \\
\text { capabilities }\end{array}$ \\
\hline Smart usage & Caching, edge computing & $\begin{array}{l}\text { Resource and context awareness, human- and community-centered traffic prioritization (e.g., smart } \\
\text { edge multicasting of on-demand streams) }\end{array}$ \\
\hline Governance & Dominance of giant operators & $\begin{array}{l}\text { Cooperation between giant and local operators (and other stakeholders) for improved return on } \\
\text { investment (Rol) }\end{array}$ \\
\hline
\end{tabular}

and remote areas are considered. Interestingly, the same low power consumption requirement supports the carbon neutrality and sustainability goals. However, technical solutions alone are not sufficient, and the involvement of other stakeholders is essential. These include local people (end users), companies, and governance that express their needs, e.g., concerning required applications, priorities, public safety connectivity, education, and health care services.

The future systems will be human-centric such that the totality, RAN and backhaul, must adapt based on end user needs even though resources might be temporally limited. People must be made aware of the limitations of the system and be trained to become smart users. They should set application priorities in a way that is good for them, and this could be dynamically adjusted, e.g., based on the time of day. Furthermore, communities must define what is affordable (device and usage plans) and sufficient such that system developers can target them.

"Have all Gs" relay and access points are not necessarily the best solution in an oasis outside the power grid since they are power hungry. Instead, simplified solutions supporting one or two technologies readily available with end user devices could be sufficient. Another justification is that in unconnected areas, people are not yet locked in any technology and they do not need compatibility with all possible systems. In addition, this results in more lightweight solutions, reduces size and the need for cooling, and is probably cheaper. Naturally, in the future, this could be based on $6 \mathrm{G}$ access technology.

In addition, it is likely that TV broadcasting and video streaming services will become future services in the unconnected areas. It calls for smart multicasting at the local 
edge, reducing the number of parallel backhaul streams to the minimum to save possibly limited backhaul and access capacity. The same, indeed, holds for all data usage. Both backhaul and access must adapt based on the load, available resources (which may be time varying, e.g., due to power availability), and priorities. They must act together to optimize the performance. Furthermore, power-saving mechanisms over the system including both relays and RAN must be included. Optimization over an area of oases that share a common backhaul is also foreseen.

Antenna selection, frequency selection, and antenna placement play a major role in required power level with link distance. Therefore, good planning and experience are needed for optimal results as well as tools that advise on this. Network planning tools are needed to design the backhaul chains, tower locations, and heights and to estimate required data rates and power, but also to utilize diffraction and large intelligent reflective surfaces if needed and seen beneficial.

Flexible, software-based modulation and coding schemes allow the implementation of power-efficient constant envelope and high-data-rate-friendly OFDMs (or other modulations) using common hardware, which reduces cost and hardware and allows the use of optimized signals for various purposes. Flexible frequency regulation allows wider bandwidth, which is an energy-efficient way to increase data rate for the backhaul in rural and remote areas, if possible from the interference point of view. This is indeed a possibility since in remote areas, some frequencies may be unused or underused. Naturally, this calls for flexibility from both radio devices and frequency regulations.

Governance, either regional or national, may play a significant role. The regulators and operators should ponder on how to benefit from the system, especially if they participate in the

\section{REFERENCES}

3 GPP (2020). Self-Organizing Networks (SON) Policy Network Resource Model (NRM) Integration Reference Point (IRP); Information Service (IS) (Release 16). TS 28.628 V16.1.0 (2020-09).

Auer, G., Giannini, V., Desset, C., Godor, I., Skillermark, P., Olsson, M., et al. (2011). How Much Energy Is Needed to Run a Wireless Network? IEEE Wireless Commun. 18, 40-49. doi:10.1109/MWC.2011.6056691

Bondalapati, P., Tiwari, A., Sahin, M. E., Tang, Q., Saraswat, S., Suryakumar, V., et al. (2020). SuperCell: A Wide-Area Coverage Solution Using High-Gain, High-Order Sectorized Antennas on Tall Towers. arXiv preprint arXiv: 2012.00161

Chaoub, A., Giordani, M., Lall, B., Bhatia, V., Kliks, A., Mendes, L., et al. (2021). 6G for Bridging the Digital Divide: Wireless Connectivity to Remote Areas. IEEE Wireless Commun., 1-9. doi:10.1109/mwc.001.2100137

Deruyck, M., Joseph, W., and Martens, L. (2014). Power Consumption Model for Macrocell and Microcell Base Stations. Trans. Emerging Tel. Tech. 25, 320-333. doi:10.1002/ett.2565

Dryancour, G. (2017). Smart Agriculture for All Farms. A CEMA Position Paper.

Edstam, J., Hansryd, J., Carpenter, S., Emanuelsson, T., Li, Y., and Zirath, H. (2017). Microwave Backhaul Evolution - Reaching beyond $100 \mathrm{GHz}$. Ericsson Technology Review.

Ericsson (2020). Ericsson Microwave Outlook. Ericsson.

Ericsson (2018). Mini Link 6000: Enabling 5G Transport Everywhere. Ericsson Microwave Outlook.

Ge, X., Cheng, H., Guizani, M., and Han, T. (2014). 5G Wireless Backhaul Networks: Challenges and Research Advances. IEEE Netw. 28, 6-11. doi:10.1109/MNET.2014.6963798 funding of the infrastructure. One obvious consequence from the improved connectivity is improved possibilities for various e-services and the nation's productivity in general. Another, less obvious, outcome is the potential for the improved connectivity of the government and other authorities in remote areas and connectivity during crises such as natural disasters. Of course, authorities have their own special requirements, e.g., related to security, that should be highlighted when the system is planned. Governance may also have an opinion regarding spectrum fees that would play a prominent role in affordability as governance is often involved when the requirements are set.

\section{DATA AVAILABILITY STATEMENT}

The original contributions presented in the study are included in the article/Supplementary Material, further inquiries can be directed to the corresponding author.

\section{AUTHOR CONTRIBUTIONS}

HS, AC, MH, AS, and VB are equal contributors.

\section{FUNDING}

This research has been supported by the Academy of Finland 6Genesis Flagship (grant 318927) and the EU's regional funding Interreg Nord via the Arctic 5G Test Networks project that is cofunded by Lapin Liitto, FI, and Region Norrbotten, SE.

GSMA Connected Society (2019). Closing the Coverage Gap - How Innovation Can Drive Rural Connectivity. White Paper.

GSMA (2021). Wireless Backhaul Evolution: Delivering Next-Generation Connectivity. A GSMA report.

Habibzadeh, M., Hassanalieragh, M., Ishikawa, A., Soyata, T., and Sharma, G. (2017). Hybrid Solar-Wind Energy Harvesting for Embedded Applications: Supercapacitor-Based System Architectures and Design Tradeoffs. IEEE Circuits Syst. Mag. 17, 29-63. doi:10.1109/mcas.2017.2757081

Handforth, C. (2019). Closing the Coverage gap; How Innovation Can Drive Rural Connectivity. GSMA Connected Society.

Hexa-X (2021). Deliverable d1.1: $6 g$ Vision, Use Cases and Key Societal Values. Call: H2020-ICT-2020-2. A HEXA-X project report. Project reference: 101015956

Huawei (2019). White Paper on Co-location System Isolation of a Sub-3 GHz Antenna and a C-Band NR Antenna. Huawei.

ITU-R (2013a). Method for point-to-area Predictions for Terrestrial Services in the Frequency Range $30 \mathrm{MHz}$ to $3000 \mathrm{MHz}$. Recommendation ITU-R P.1546-6.

ITU-R (2013b). Propagation by Diffraction. Recommendation ITU-R P.526-13.

Jaber, M., Imran, M. A., Tafazolli, R., and Tukmanov, A. (2016). 5g Backhaul Challenges and Emerging Research Directions: A Survey. IEEE Access 4, 1743-1766. doi:10.1109/ACCESS.2016.2556011

Khaturia, M., Jha, P., and Karandikar, A. (2020). Connecting the Unconnected: Toward Frugal 5G Network Architecture and Standardization. IEEE Comm. Stand. Mag. 4, 64-71. doi:10.1109/MCOMSTD.001.1900006

Kumar, A., Karandikar, A., Naik, G., Khaturia, M., Saha, S., Arora, M., et al. (2016). Toward Enabling Broadband for a Billion Plus Population with TV White Spaces. IEEE Commun. Mag. 54, 28-34. doi:10.1109/mcom.2016.7509375

Kusuma, J., and Boch, E. (2021). Improving Rural Connectivity Coverage Using Diffractive Non-line of Sight (NLOS) Wireless Backhaul. WWRF45. 
Latva-aho, M., and Leppänen, K. (2019). "Key Drivers and Research Challenges for 6G Ubiquitous Wireless Intelligence," in $6 G$ Research Visions. Editors M LatvaAho and K Leppänen (University of Oulu), 1.

Monti, P., Tombaz, S., Wosinska, L., and Zander, J. (2012). "Mobile Backhaul in Heterogeneous Network Deployments: Technology Options and Power Consumption," in 2012 14th International Conference on Transparent Optical Networks (ICTON), Coventry, UK, July 2-5, 2012, 1-7. doi:10.1109/ICTON.2012.6253839

Nikandish, G., Staszewski, R. B., and Zhu, A. (2020). Breaking the Bandwidth Limit: A Review of Broadband Doherty Power Amplifier Design for 5G. IEEE Microwave 21, 57-75. doi:10.1109/MMM.2019.2963607

Omslandseter, R. O., Jiao, L., and Haglund, M. A. (2018). "Field Measurements and Parameter Calibrations of Propagation Model for Digital Audio Broadcasting in Norway," in 2018 IEEE 88th Vehicular Technology Conference (VTC-Fall), Chicago, United States, August 27-30, 2018, 1-6. doi:10.1109/VTCFall.2018.8690746

Özdogan, Ö., Björnson, E., and Larsson, E. G. (2019). Intelligent Reflecting Surfaces: Physics, Propagation, and Pathloss Modeling. IEEE Wireless Commun. Lett. 9, 581-585. doi:10.1109/LWC.2019.2960779

Pärssinen, A., Alouini, M., Berg, M., Kuerner, T., Kyösti, P., Leinonen, M., et al. (2020). "White Paper on RF Enabling 6G-Opportunities and Challenges from Technology to Spectrum," in $6 G$ Research Visions (University of Oulu), 13.

Pérez-Peña, S., Castiella-Fernández, M., Velasco-de-la-Fuente, P., Riera, J. M., Burgos-García, M., García-del-Pino, P., et al. (2020). "Development of Measurement and Modeling Procedures of Diffractive Near-LOS Wireless Links," in 2020 XXXIIIrd General Assembly and Scientific Symposium of the International Union of Radio Science, Rome, Italy, August 29-September 5, 2020, 1-4. doi:10.23919/URSIGASS49373.2020.9232262

Polese, M., Giordani, M., Zugno, T., Roy, A., Goyal, S., Castor, D., et al. (2020). Integrated Access and Backhaul in $5 \mathrm{~g}$ Mmwave Networks: Potential and Challenges. IEEE Commun. Mag. 58, 62-68. doi:10.1109/MCOM.001.1900346

Racom (2021). RAy - Microwave Link. Product leaflet, Downloaded Feb 2021. Racom.

Rademacher, M., Kretschmer, M., and Jonas, K. (20132014). "Exploiting IEEE802.11n MIMO Technology for Cost-Effective Broadband Back-Hauling," in e-Infrastructure and e-Services for Developing Countries. 5th International Conference, AFRICOMM 2013, Blantyre, Malawi, November 25-27, 2013. Editor T Bissyandé (Springer International Publishing), 135. Revised selected papers.

Saarnisaari, H., Dixit, S., Alouini, M.-S., Chaoub, A., Giordani, M., Kliks, A., et al. (2020). "6G white Paper on Connectivity for Remote Areas," in 6G Research Visions, Levi, Finland, November 26-27, 2020 (University of Oulu), 5.

Saarnisaari, H. (2014). "Estimation and Use of Load Threshold for Energy Saving in Wireless Networks," in 1st International Conference on 5G for Ubiquitous Connectivity (5GU 2014). doi:10.4108/icst.5gu.2014.258082

Saarnisaari, H., and Lima, C. (2020). Application of 5G New Radio for Satellite Links with Low Peak-to-average Power Ratios. Int. J. Satell Commun. Netw. 39, 445-454. doi:10.1002/sat.1378
Shaik, P., Singya, P., and Bhatia, V. (2019). On Impact of Imperfect CSI Over Hexagonal QAM for TAS/MRC-MIMO Cooperative Relay Network. IEEE Commun. Lett. 23 (10), 1721-1724. doi:10.1109/lcomm.2019.2931433

Singya, P., Kumar, N., and Bhatia, V. (2017). Mitigating NLD for Wireless Networks: Effect of Nonlinear Power Amplifiers on Future Wireless Communication Networks. IEEE Microw. Mag. 18 (5), 73-90. doi:10.1109/ MMM.2017.2691423

Singya, P., Shaik, P., Kumar, N., Bhatia, V., and Alouini, M.-S. (2021). A Survey on HigherOrder QAM Constellations: Technical Challenges, Recent Advances, and Future Trends. IEEE Open J. Commun. Soc. 2, 617-655. doi:10.1109/ojcoms.2021.3067384

Tezergil, B., and Onur, E. (2021). Wireless Backhaul in 5G and Beyond: Issues, Challenges and Opportunities. arXiv preprint arXiv:2103.08234.

Tipmongkolsilp, O., Zaghloul, S., and Jukan, A. (2011). The Evolution of Cellular Backhaul Technologies: Current Issues and Future Trends. IEEE Commun. Surv. Tutorials 13, 97-113. doi:10.1109/SURV.2011.040610.00039

Tombaz, S., Monti, P., Farias, F., Fiorani, M., Wosinska, L., and Zander, J. (2014). "Is Backhaul Becoming a Bottleneck for green Wireless Access Networks," in 2014 IEEE International Conference on Communications (ICC), Sydney, Australia, June 10-14, 2014, 4029-4035. doi:10.1109/ICC.2014.6883951

Vasjanov, A., and Barzdenas, V. (2020). A Review of Advanced CMOS RF Power Amplifier Architecture Trends for Low Power 5G Wireless Networks. Electronics 7, 271. doi:10.3390/electronics7110271

Wu, Q., and Zhang, R. (2019). Towards Smart and Reconfigurable Environment: Intelligent Reflecting Surface Aided Wireless Network. IEEE Commun. Mag. 58, 106-112. doi:10.1109/MCOM.001.1900107

Yaacoub, E., and Alouini, M.-S. (2020). A Key 6G Challenge and OpportunityConnecting the Base of the Pyramid: A Survey on Rural Connectivity. Proc. IEEE 108, 533-582. doi:10.1109/jproc.2020.2976703

Conflict of Interest: The authors declare that the research was conducted in the absence of any commercial or financial relationships that could be construed as a potential conflict of interest.

Publisher's Note: All claims expressed in this article are solely those of the authors and do not necessarily represent those of their affiliated organizations, or those of the publisher, the editors and the reviewers. Any product that may be evaluated in this article, or claim that may be made by its manufacturer, is not guaranteed or endorsed by the publisher.

Copyright $\odot 2021$ Saarnisaari, Chaoub, Heikkilä, Singhal and Bhatia. This is an open-access article distributed under the terms of the Creative Commons Attribution License (CC BY). The use, distribution or reproduction in other forums is permitted, provided the original author(s) and the copyright owner(s) are credited and that the original publication in this journal is cited, in accordance with accepted academic practice. No use, distribution or reproduction is permitted which does not comply with these terms. 